\title{
Impact of Pharmacist-Led Cognitive Behavioural Therapy for Chronic Insomnia
}

Joshua Nurkowski, PharmD (candidate); College of Pharmacy and Nutrition, University of Saskatchewan, Saskatoon, SK

Habiba Elshorbagy, BSP; Saskatchewan Health Authority, Saskatoon, SK

Katelyn Halpape, BSP, ACPR, PharmD, BCPP; College of Pharmacy and Nutrition, University of Saskatchewan, Saskatoon, SK

Karen Jensen, BSP, MSC (Pharm); College of Pharmacy and Nutrition, University of Saskatchewan, Saskatoon, SK

Darcy A Lamb, BSP, ACPR, MSc (Pharm); Saskatchewan Health Authority, Saskatoon, SK

Eric Landry, BSP, ACPR; College of Pharmacy and Nutrition, University of Saskatchewan, Saskatoon, SK

Alfred Remillard, BSC, BSC (Pharm), PharmD; College of Pharmacy and Nutrition, University of Saskatchewan, Saskatoon, SK

Derek Jorgenson, BSP, PharmD, FCSHP; College of Pharmacy and Nutrition, University of Saskatchewan, Saskatoon, SK

\begin{abstract}
Background: Chronic insomnia is a common medical condition that negatively impacts quality of life and daytime function. Access to the first-line treatment for insomnia, cognitive behavioural therapy (CBT-i), is limited. Pharmacists are well positioned to provide this service, but evidence regarding pharmacist delivered CBT-i is sparse. The aim of this study was to evaluate the effectiveness of CBT-i delivered by pharmacists practicing in an outpatient clinic setting.

Methods: This study was a retrospective chart audit of adult patients with chronic insomnia who received CBT-i from a pharmacist at one of two outpatient clinics in Canada. The primary endpoints were the differences between patient self-reported sleep diary parameters and utilization of hypnotic medications before and after CBT-i was delivered. The differences in patient reported sleep parameters were compared using Wilcoxon Signed Rank test and paired samples t-test and changes in hypnotic utilization was compared using McNemar Chi-square test.

Results: 183 patients were referred for CBT-i and attended an initial appointment with a pharmacist. Of these, 105 did not receive the CBT-i. This resulted in 78 patients who met the inclusion criteria. Changes in sleep diary parameters were all statistically significantly improved after patients received CBT-i, except for total sleep time. Hypnotic medication use was also reduced. At baseline, $71.8 \%$ $(n=56 / 78)$ of patients were taking one or more hypnotic medications compared to $52.6 \%(n=41 / 78)$ after CBT-i ( $p=0.0003)$.

Discussion: The results of this study provide preliminary evidence that pharmacists working in an outpatient clinic setting may be able to effectively deliver CBT-i for patients with chronic insomnia. The external validity of these results is limited by the observational study design and the inclusion of pharmacists practicing in outpatient clinics, which is not the setting where most pharmacists currently practice.

Conclusion: This observational study found improvements in sleep quality and efficiency, as well as, a reduction in hypnotic medication use, in patients who received CBT-i from pharmacists practicing in an outpatient clinic setting. Future randomized, controlled trials should evaluate the impact of CBT-i in a larger sample of patients, provided by pharmacists practicing in both outpatient clinics and community pharmacies.
\end{abstract}

Keywords: insomnia, primary care, cognitive behavioural therapy, pharmacy practice

\section{Introduction}

Chronic insomnia is defined as the frequent inability to initiate and/or maintain sleep, experiencing non-restorative sleep, and/or early morning awakenings lasting greater than three months. ${ }^{1-3}$ Chronic insomnia can also cause a number of daytime symptoms such as fatigue, sleepiness, poor concentration, and irritability. ${ }^{1}$ Chronic insomnia can even impact the physical and mental health of individuals by increasing the risk of cardiovascular disease, diabetes, obesity, and depression. ${ }^{2}$ This condition also negatively impacts society through decreased productivity and increased absenteeism from school and work. ${ }^{4}$

Corresponding author: Derek Jorgenson, BSP, PharmD, FCSHP Professor, College of Pharmacy and Nutrition

University of Saskatchewan

104 Clinic Place, Saskatoon, SK S7N $2 Z 4$

(p) 306-966-2009 (f) 306-966-6377
Several professional organizations, including the American Academy of Sleep Medicine, the American College of Physicians, Toward Optimized Practice (Canada) and the European Sleep Research Society, recommend Cognitive Behavioural Therapy (CBT-i) as the first-line treatment for chronic insomnia. ${ }^{5-9}$ However, in many cases, patients seeking help for this condition are initiated on a hypnotic medication during their initial encounter with a healthcare professional. ${ }^{1,10}$ For instance, a study in Australia found that community pharmacists recommended an over-the-counter sleep aid as first-line therapy in $96 \%$ of sleep complaint cases. ${ }^{11}$

The evidence for use of hypnotic medications, such as benzodiazepines and z-drugs (i.e., zopiclone, zolpidem), is limited to short-term treatment (i.e., less than four weeks) and these medications are not indicated for chronic use. ${ }^{7,12}$ Patients can also develop physiological and psychological dependence to hypnotic medications if they are used on a continuous basis. ${ }^{1}$ Additionally, hypnotic medications have high rates of adverse 
effects including drowsiness and fatigue, which can result in falls and motor vehicle accidents. ${ }^{13,14}$ The risks of hypnotics, along with their limited long term effectiveness, justify the importance of focusing on CBT-i as the first-line treatment for chronic insomnia.

The provision of CBT-i includes offering patients an individualized combination of cognitive, behavioural, and educational interventions to treat the chronic insomnia. CBT-i consists of six components that are typically completed over an average of 4-10 sessions with a trained health professional. These components include stimulus control, sleep restriction, sleep hygiene, cognitive restructuring, relaxation training, and other stress reduction techniques. ${ }^{15}$ The efficacy of CBT-i has been demonstrated in many clinical trials and systematic reviews and includes significant improvements in both sleep quality and quantity. ${ }^{16-22}$ Despite the negative impact that comorbid medical and psychiatric conditions can have on sleep disorders, CBT-i has been shown to be efficacious in patients with stable depression or anxiety, post-traumatic stress disorder, substance use disorder, and chronic pain. ${ }^{23-26} \mathrm{~A}$ study by Jacobs et al. concluded that CBT-i was more efficacious than pharmacotherapy alone and that pharmacotherapy in addition to CBT-i provided no added long term benefit. ${ }^{27}$ Unlike hypnotic medications, which have evidence for only short-term effectiveness, improvements in sleep have been shown to be sustained for up to 24 months after CBT-i has been completed. ${ }^{28}$

The primary limitation of CBT-i, when delivered in-person by a health care provider, is that it is a service that requires significant time, specialized training, and numerous follow-up patient interactions. ${ }^{29}$ Consequently, there is a limited number of health professionals who provide CBT-i, despite the evidence for its effectiveness. ${ }^{30}$ Previous studies have found that CBT-i can be effectively delivered by non-sleep specialists, such as nurses, psychologists, therapists, and social workers. ${ }^{18,25,31,32}$ Unfortunately, patient access to this service remains limited and the need to train more primary health workers to provide CBT-i has been discussed widely in the literature. ${ }^{29,33,34}$

Pharmacists are well positioned to provide CBT-i due to their highly accessible position in the health systems of many countries. Historically, pharmacists have been commonly involved in providing education and follow-up care related to the use of hypnotics. ${ }^{35,36}$ A review by the Canadian Agency for Drugs and Technologies in Health (CADTH) reported that $62 \%$ of surveyed pharmacists discuss behavioural interventions (e.g. sleep hygiene, relaxation training, stimulus control) during their prescription counselling sessions with patients starting treatment with hypnotics. ${ }^{10}$ However, there is very limited published research on pharmacists providing CBT-i. One small observational study reported data from 11 patients in Canada who had improvements in some sleep parameters after receiving CBT-i from pharmacists. ${ }^{37}$ A slightly larger study by Fuller et al. $(n=41)$ measured the impact of pharmacist- provided behavioural interventions for insomnia patients; however, the intervention was comprised of only two of the six components of CBT-i (i.e., stimulus control and sleep restriction). ${ }^{38}$ The aim of this study was to evaluate the effectiveness of CBT-i delivered by pharmacists practicing within an outpatient clinic setting in Canada. The primary endpoints were the differences between patient self-reported sleep diary parameters and utilization of any hypnotic medication before and after the CBT-i service was delivered.

\section{METHODS}

This study was a retrospective chart audit of adult patients $(\geq 18$ years) who received CBT-i from a pharmacist at one of two outpatient clinics in Saskatoon, Saskatchewan (Canada), the Medication Assessment Centre (MAC) and West Winds Primary Health Centre (WWPHC). Both clinics have a pharmacist on staff who offers individual patient consultations, in an office-based setting, on referral from a health professional or from patients directly. These clinics do not include a dispensary and the pharmacists do not fill prescriptions.

Pharmacists at the MAC and WWPHC received training to provide $\mathrm{CBT}-\mathrm{i}$ by attending a professional development program called PharmaZzz, which was offered by the College of Pharmacy and Nutrition at the University of Saskatchewan. Pharmacists enrolled in the PharmaZzz program were provided with a self-study manual, which explained how to provide CBTi and included a stepwise approach to providing the service. Participants were also provided with a supply of workbooks that could be given to patients during CBT-i consultations, which provided detailed insomnia patient education and a sleep diary. The self-study manual and patient workbook were developed from existing models for CBT-i and adapted for pharmacy practice by psychiatry faculty at the University of Saskatchewan. After completing the self-study manual, pharmacists were required to attend a one day in person workshop in which local physician and pharmacist sleep experts provided additional educational content on the pathophysiology of sleep disorders, CBT-i theory and application, patient assessment, tapering of hypnotics, and interpretation of sleep logs. Participants in the one-day workshop also had the opportunity to role play and receive feedback from workshop facilitators to complete the CBT-i training program.

Chronic insomnia is commonly clinically assessed using patient self-reported sleep diary parameters, which are typically collected during CBT-i consultations. ${ }^{3}$ These parameters include: the time it takes for an individual to fall asleep (known as sleep onset latency or SOL); the sum of awake time occurring between initial sleep onset and the final morning awakening (known as wake after sleep onset ,Ikor WASO); the total number of hours of sleep (known as total sleep time or TST); and the proportion of time spent in bed actually asleep (known as sleep efficiency or SE). ${ }^{4}$ The severity of chronic insomnia, from the patient's perspective, can also be assessed using the 
validated Insomnia Severity Index (ISI) questionnaire, which includes a variety of patient-reported sleep measures. ${ }^{5}$ Guidelines suggest that the goals for chronic insomnia management are to improve sleep quality and duration by reducing both SOL and WASO to less than 30 minutes and increasing SE to greater than $80-85 \% .^{5}$ The primary endpoints of this study were the differences between all of these patient self-reported sleep diary parameters and utilization of hypnotic medications before and after the CBT-i service was delivered by the two pharmacists at WWPHC and the MAC.

All patients, 18 years and older, who received CBT-i from a pharmacist at either the MAC or WWPHC between August 2016 and June 2019 were included in this study. Patients were excluded if they had an inadequately controlled medical condition that would make them a poor candidate for CBT-i (e.g., generalized anxiety disorder). Chart data of patients who met the inclusion criteria were extracted from the electronic medical records (EMR) at the MAC and WWPHC in July 2019. Data extraction was performed by an undergraduate research student at the University of Saskatchewan who was not part of the clinical team at either WWPHC or the MAC. The following patient self-reported sleep diary parameters were collected, both pre and post implementation of the CBT-i service, (if they were documented by the pharmacist in the EMR): SOL, WASO, the total number of nighttime awakenings, total time in bed (TIB), TST, SE (calculated by dividing the TST by the TIB), and the ISI score. Pre-implementation parameters were defined as those collected by the pharmacist at the initial consultation, before any cognitive or behavioral interventions were implemented. Post-implementation parameters were defined as those collected by the pharmacist at the most recent consultation, after all planned cognitive and behavioral interventions were implemented.

Hypnotic medication utilization was measured by comparing the baseline patient medication lists in the EMR, compiled by the pharmacist at the initial CBT-i appointment, with the medication lists compiled at the final follow-up CBT-i appointment. Any medication that the patient reported to be taking for sleep was included, even if the medication was not officially indicated for the treatment of insomnia. This includes prescription/non-prescription medications, natural products, or supplements. If there were any differences between the medication lists (e.g. hypnotic medication additions, discontinuations, or dose changes) the charts were reviewed to determine if the changes were directly related to a recommendation made by the pharmacist (i.e., if there was a documented recommendation made by the pharmacist in the EMR). Only changes to hypnotic medication utilization that were specifically recommended by the pharmacists were included in the analysis.

The differences in patient reported SOL, WASO, total number of nighttime awakenings, total TIB, SE, and ISI scores pre and post CBT-i were compared using the Wilcoxon Signed Rank test.
Changes in TST were compared using the paired samples t-test and changes in hypnotic utilization were compared using the McNemar Chi-square test. Data analyses were completed using IBM SPSS Statistics software (Version 25.0. Armonk, NY: IBM Corp). This research protocol was approved by the University of Saskatchewan research ethics board.

\section{RESULTS}

A total of 183 patients were referred for CBT-i and attended an initial appointment with a pharmacist at either the MAC or WWPHC. However, 105 of these patients did not receive the CBT-i service for one or more of the following reasons: pharmacist decided the patient was not a good candidate for CBT- $i(n=19 / 105,18.1 \%)$, the patient refused the CBT- $i$ intervention after learning what it entailed ( $n=32 / 105,30.5 \%)$, and the patient did not return for follow-up for unknown reasons $(n=54 / 105,51.4 \%)$. This resulted in 78 patients who received the CBT-i intervention, met the inclusion criteria, and were included in the analyses (Figure 1). The majority of patients included in this study $(\mathbf{7 1 . 8 \% )}$ ) were taking one or more hypnotic medication at baseline and the most commonly used hypnotics were zopiclone (53.6\%), trazodone $(12.5 \%)$, and melatonin (10.7\%) - Table 1.

The mean number of documented, pharmacist-delivered, CBT-i appointments was 5.1 per patient (range of 3-13). The patient self-reported sleep diary parameters, along with the ISI scores, were all statistically significantly improved after patients received CBT-i, except for the TST (Table 2). Hypnotic medication use was also statistically significantly reduced after patients completed CBT-i. At baseline, 71.8\% ( $n=56 / 78)$ of patients were taking one or more hypnotic medication compared with $52.6 \%(n=41 / 78)$ after CBT-i $(p=0.0003)$. Of the 41 patients who remained on one or more hypnotic medication after receiving CBT-i, 66.1\% ( $n=27 / 41)$ were taking a reduced dose.

\section{Discussion}

Previous research has consistently found that CBT-i can be effectively delivered by a broad range of non-sleep specialist health care professionals, such as nurses, psychologists, therapists, and social workers. ${ }^{18,25,31,32}$ The results of this nonrandomized, observational study add unique and valuable data to the existing literature by providing preliminary evidence that pharmacists working in an outpatient clinic setting may also be able to effectively deliver CBT-i for patients with chronic insomnia.

The improvements in sleep parameters observed in this study are similar to those reported in previous studies in which other non-sleep specialist health professionals performed the CBT-i. ${ }^{18,25,31,32}$ This offers some confidence in the validity of these results since the CBT-i provided by the pharmacists in this study had a similar effect as was reported in previous research not involving pharmacists. Similarly, the range of patient appointments that was required for the pharmacists to deliver 
the service in this study (i.e., 3-13 appointments) was similar to findings in previous research of other health professionals who provided CBT-i (i.e., 4-10 appointments). ${ }^{18,25,31,32}$

The improvements in sleep quality and efficiency reported in this study also appear to be clinically meaningful. Sleep onset latency (SOL) was reduced to less than 30 minutes and SE improved to over $85 \%$, which are both considered successful treatment targets defined in insomnia guidelines. ${ }^{5}$ It may be more useful to interpret these data by simply considering the raw numbers. At baseline, on average, patients were taking approximately 48 minutes to fall asleep and were awake for over 60 minutes during the night. After CBT-i, both of these parameters were reduced by almost $50 \%$, which is an improvement that patients would likely find to be meaningful (Table 2). It is not surprising that total sleep time was unchanged in this study, as previous research has reported a similar finding. ${ }^{18,25,31,32}$ Individuals diagnosed with chronic insomnia typically have difficulty falling asleep and/or do not sleep efficiently (i.e., wake up frequently, do not have restorative sleep), despite often getting the recommended or close to recommended total quantity of sleep throughout an entire day.

The improvements observed in the ISI scores provide additional evidence of the clinical importance of the results of this study. The previously validated ISI is frequently used in research, and in clinical practice, to assess and quantify response to treatment. ${ }^{39}$ The minimal clinically important difference for the ISI score is considered to be a change of at least 7 points and a change greater than 9 points is considered to be a "marked improvement". ${ }^{39}$ The results of this study found that mean ISI scores improved by 10.3 points, from a baseline of 18.2 (defined as 'moderately severe insomnia') to 7.9 (defined as 'subthreshold insomnia'), suggesting that, on average, patients in this study had a marked improvement in their chronic insomnia symptoms.

This study also found a statistically significant reduction in overall hypnotic medication use. It was not a pre-defined study endpoint to perform separate statistical analyses on changes related to each different hypnotic agent; however, zopiclone was, by far, the most commonly utilized medication in this study. This is an important finding as this suggests that the patients were sleeping better despite taking fewer medications after CBT-i. Hypnotic usage was reduced from $71.8 \%$ to $52.6 \%$ of patients, an absolute reduction of $19.2 \%$. In addition, almost two-thirds of patients, who remained on a hypnotic after CBT-i was completed, were taking a lower dose.

It is important to note the large proportion of patients who were referred for $\mathrm{CBT}-\mathrm{i}$, attended an initial appointment, but did not actually receive the service ( $n=105 / 183,57.3 \%)$. This finding is not surprising, as many patients may not be suitable candidates for CBT-i (e.g., poorly controlled psychiatric comorbidities, secondary insomnia, contraindication for CBT-i) and often this may not be identified until after the initial CBT-i appointment. Additionally, the cognitive and behavioural interventions required of patients undergoing CBT-i are challenging and require significant patient motivation, determination, and commitment, thus, it is understandable that many patients may decline CBT-i after they learn about what it entails during the initial appointment, as was observed in this study.

There are some significant limitations to this study that suggest the results should be applied into practice with caution and interpreted as hypothesis generating. Most importantly is the non-randomized, uncontrolled, observational study design, which was selected intentionally to determine if there was an initial signal that there may be some value to pharmacist-led CBT-i. One of the primary endpoints, changes in sleep diary parameters, relies completely on patient self-reporting of data. Although these endpoints are commonly used in research related to chronic insomnia, there is no way to verify the accuracy or reliability of the patient's sleep diaries. In addition, this study evaluated a service provided by only two pharmacists who both practice in a non-dispensing, outpatient, primary care clinic setting. It is not known if these results would be similar if provided by a large sample of pharmacists in a community pharmacy setting. Finally, this study did not collect data on long-term consequences of chronic insomnia (i.e., performance/attendance at work/school) and only reported changes in patient self-reported sleep parameters and hypnotic medication usage.

\section{Conclusions}

Pharmacist-led CBT-i, performed within two non-dispensing, outpatient clinics in Canada, resulted in statistically significant and clinically meaningful improvements in sleep quality and efficiency, along with reductions in hypnotic medication utilization. This is important new information that provides a strong rationale for larger scale studies to be completed to further evaluate the role of pharmacists providing CBT-i. Additional randomized, controlled studies should be performed to confirm these results in a much larger sample of patients, recruited from multiple locations, and including pharmacists practicing in both outpatient clinic and community pharmacy settings.

Financial Acknowledgements: This study was financed with operating funds from the Medication Assessment Centre. The Medication Assessment Centre is funded by unrestricted educational grants from the University of Saskatchewan and the Saskatchewan Ministry of Health.

Conflict of interest statement: The authors have no conflicts of interest to report. 


\section{References}

1. Robotham D. Sleep as a public health concern: Insomnia and mental health. J Public Ment Health. 2011. doi:10.1108/17465721111188250

2. Luyster FS, Strollo PJ, Zee PC, Walsh JK. Sleep: A Health Imperative. Sleep. 2012;35(6):727-734.

doi:10.5665/sleep.1846

3. Sleep-Wake Disorders: Diagnostic and Statistical Manual of Mental Disorders: DSM-5. $5^{\text {th }}$ ed. Arlington VA:

American Psychiatric Publishing; 2013.

4. Daley M, Morin CM, Leblanc M, Grégoire J-P, Savard J. The Economic Burden of Insomnia: Direct and Indirect Costs for Individuals with Insomnia Syndrome, Insomnia Symptoms, and Good Sleepers. Sleep. 2009;32(1):55-64.

5. Schutte-Rodin SL, Broch L, Buysee D, Dorsey C, Sateia M. Clinical guideline for the evaluation and management of chronic insomnia in adults. J Clin Sleep Med. 2008;4(5):487-504. doi:10.5664/jcsm.27286

6. Qaseem A, Kansagara D, Forciea MA, Cooke M, Denberg TD. Management of Chronic Insomnia Disorder in Adults: A Clinical Practice Guideline From the American College of Physicians. Ann Intern Med. 2016;165(2):125. doi:10.7326/M15-2175

7. Sateia MJ, Buysse DJ, Krystal AD, Neubauer DN, Heald JL. Clinical Practice Guideline for the Pharmacologic Treatment of Chronic Insomnia in Adults: An American Academy of Sleep Medicine Clinical Practice Guideline. J Clin Sleep Med. 2017;13(2):307-349.

doi: $10.5664 / j c s m .6470$

8. Assessment to Management of Adult Insomnia: Clinical Practice Guideline . Edmonton AB: Toward Optimized Practice; 2015.

9. Riemann D, Baglioni C, Bassetti C, et al. European guideline for the diagnosis and treatment of insomnia. $J$ Sleep Res. 2017;26(6):675-700. doi:10.1111/jsr.12594

10. Crain J, Raj M, Garland S, Jones S, Farah B. Current Practice Analysis: Interventions for Insomnia Disorder. CADTH. 2017.

11. Kippist C, Wong K, Bartlett D, Saini B. How do pharmacists respond to complaints of acute insomnia? A simulated patient study. Int J Clin Pharm. 2011;33(2):237-245. doi:10.1007/s11096-011-9482-5

12. Conn DK, Hogan DB, Amdam L, et al. Canadian Guidelines on Benzodiazepine Receptor Agonist Use Disorder Among Older Adults. Can Geriatr Soc. 2020;23(1):116-122.

13. Brandt J, Leong C. Benzodiazepines and Z-Drugs: An Updated Review of Major Adverse Outcomes Reported on in Epidemiologic Research. Drugs $R D$. 2017;17(4):493-507. doi:10.1007/s40268-017-0207-7

14. Glass J, Lanctôt KL, Herrmann N, Sproule BA, Busto UE. Sedative hypnotics in older people with insomnia: Metaanalysis of risks and benefits. Br Med J. 2005. doi:10.1136/bmj.38623.768588.47

15. Siebern AT, Manber R. New developments in cognitive behavioral therapy as the first-line treatment of insomnia. Psychol Res Behav Manag. 2011;4:21-28. doi:10.2147/prbm.s10041
16. Trauer JM, Qian MY, Doyle JS, Rajaratnam SMW Cunnington D. Cognitive Behavioral Therapy for Chronic Insomnia. Ann Intern Med. 2015;163(3):191. doi:10.7326/M14-2841

17. Morin CM, Culbert JP, Schwartz SM. Nonpharmacological interventions for insomnia: A metaanalysis of treatment efficacy. Am J Psychiatry. 1994;151(8):1172-1180. doi:10.1176/ajp.151.8.1172

18. Wang MY, Wang SY, Tsai PS. Cognitive behavioural therapy for primary insomnia: A systematic review. J Adv Nurs. 2005;50(5):553-564. doi:10.1111/j.13652648.2005.03433.x

19. Okajima I, Komada Y, Inoue Y. A meta-analysis on the treatment effectiveness of cognitive behavioral therapy for primary insomnia. Sleep Biol Rhythms. 2011;9(1):2434. doi:10.1111/j.1479-8425.2010.00481.x

20. Brasure M, Fuchs E, MacDonald R, et al. Psychological and Behavioral Interventions for Managing Insomnia Disorder: An Evidence Report for a Clinical Practice Guideline by the American College of Physicians. Ann Intern Med. 2016;165(2):113. doi:10.7326/M15-1782

21. Koffel EA, Koffel JB, Gehrman PR. A meta-analysis of group cognitive behavioral therapy for insomnia. Sleep Med Rev. 2014;19:6-16. doi:10.1016/j.smrv.2014.05.001

22. Jansson M, Linton SJ. Cognitive-Behavioral Group Therapy as an Early Intervention for Insomnia: A Randomized Controlled Trial. J Occup Rehabil. 2005;15(2). doi:10.1007/s10926-005-1217-9

23. Okajima I, Inoue Y. Efficacy of cognitive behavioral therapy for comorbid insomnia: a meta-analysis. Sleep Biol Rhythms. 2018;16(1):21-35. doi:10.1007/s41105017-0124-8

24. Currie SR, Wilson KG, Pontefract AJ, DeLaplante L. Cognitive-behavioral treatment of insomnia secondary to chronic pain. J Consult Clin Psychol. 2000;68(3):407416. doi:10.1037/0022-006X.68.3.407

25. Sadler P, McLaren S, Klein B, Harvey J, Jenkins $M$. Cognitive behavior therapy for older adults with insomnia and depression: A randomized controlled trial in community mental health services. Sleep. 2018;41(8). doi:10.1093/sleep/zsy104

26. Taylor DJ, Pruiksma KE. Cognitive and behavioural therapy for insomnia (CBT-I) in psychiatric populations: A systematic review. Int Rev Psychiatry. 2014;26(2):205213. doi:10.3109/09540261.2014.902808

27. Jacobs GD, Pace-Schott EF, Stickgold R, Otto MW. Cognitive behavior therapy and pharmacotherapy for insomnia: A randomized controlled trial and direct comparison. Arch Intern Med. 2004;164(17):1888-1896. doi:10.1001/archinte.164.17.1888

28. Morin CM, Colecchi C, Stone J, Sood R, Brink D. Behavioral and pharmacological therapies for late-life insomnia: A randomized controlled trial. J Am Med Assoc. 1999;281(11):991-999. doi:10.1001/jama.281.11.991

29. Gratzer D, Goldbloom D. Making Evidence-Based Psychotherapy More Accessible in Canada. Can J Psychiatry. 2016;61(10):618-623. doi:10.1177/0706743716642416 
30. Williams J, Roth A, Vatthauer K, McCrae CS. Cognitive behavioral treatment of Insomnia. Chest. 2013;143(2):554-565. doi:10.1378/chest.12-0731

31. Sandlund C, Hetta J, Nilsson GH, Ekstedt M, Westman J. Improving insomnia in primary care patients: $A$ randomized controlled trial of nurse-led group treatment. Int J Nurs Stud. 2017;72:30-41. doi:10.1016/j.ijnurstu.2017.03.007

32. Edinger JD, Wohlgemuth WK, Radtke RA, Coffman CJ, Carney CE. Dose-response effects of cognitivebehavioral insomnia therapy: A randomized clinical trial. Sleep. 2007;30(2):203-212. doi:10.1093/sleep/30.2.203

33. Cheung JMY, Ji XW, Morin CM. Cognitive Behavioral Therapies for Insomnia and Hypnotic Medications: Considerations and Controversies. Sleep Med Clin. 2019;14(2):253-265. doi:10.1016/j.jsmc.2019.01.006

34. Perlis ML, Smith MT, Cacialli DO, Nowakowski S, Orff H. On the comparability of pharmacotherapy and behavior therapy for chronic insomnia: Commentary and implications. J Psychosom Res. 2003;54(1):51-59. doi:10.1016/S0022-3999(02)00547-0

35. Kuntz JL, Kouch L, Christian D, Hu W, Peterson PL. Patient Education and Pharmacist Consultation Influence on Nonbenzodiazepine Sedative Medication Deprescribing Success for Older Adults. Perm J. 2019;23:18-161. doi:10.7812/TPP/18-161

36. Fuller JM, Wong KK, Krass I, Grunstein R, Saini B. Sleep disorders screening, sleep health awareness, and patient follow-up by community pharmacists in Australia. Patient Educ Couns. 2011;83(3):325-335. doi:10.1016/j.pec.2011.05.004

37. Remillard F, Jensen K, Regier L, Trifa J, Edgington L. PharmaZzz: feasibility and impact of pharmacistdelivered cognitive behavioural therapy for chronic insomnia. Can Pharm J. 2017;150(4):S42. doi:10.1177/1715163517719855

38. Fuller JM, Wong KK, Hoyos C, Krass I, Saini B. Dispensing good sleep health behaviours not pills - a clusterrandomized controlled trial to test the feasibility and efficacy of pharmacist-provided brief behavioural treatment for insomnia. J Sleep Res. 2016;25(1):104-115. doi:10.1111/jsr.12328

39. Morin CM, Belleville G, Bélanger L, Ivers $H$. The insomnia severity index: Psychometric indicators to detect insomnia cases and evaluate treatment response. Sleep. 2011;34(5):601-608. doi:10.1093/sleep/34.5.601 


\begin{tabular}{|l|c|c|}
\hline \multicolumn{2}{|c|}{ Table 1: Baseline Participant Demographics (n=78) } \\
\hline Female gender - \# (\%) & & $64(82.1)$ \\
\hline Mean age - yr & & 54.9 \\
\hline Mean total medication count & & 56 (71.8) \\
\hline Hypnotic use (any) - \# (\%) & Zopiclone & $30(53.6)$ \\
\hline Types of hypnotics used - \# (\%)* & Trazodone & $7(12.5)$ \\
\hline & Melatonin & $6(10.7)$ \\
\hline & Benzodiazepine & $5(8.9)$ \\
\hline & Quetiapine & $5(8.9)$ \\
\hline & Tricyclic antidepressant & $4(7.1)$ \\
\hline & Dimenhydrinate & $2(3.6)$ \\
\hline Alcohol (6 hr before bed) - \# (\%) & Diphenhydramine & $2(3.6)$ \\
\hline Current smoking - \# (\%) & Other^ & $3(5.4)$ \\
\hline Caffeine (6 hr before bed) - \# (\%) & & $11(14.1)$ \\
\hline
\end{tabular}

* Percentages add up to more than 100 because some patients were taking multiple hypnotics

$\wedge$ Hydroxyzine, mirtazapine, cannabis

Table 2 - Patient Self-Reported Sleep Diary Parameters

\begin{tabular}{|l|c|c|c|c|}
\hline Parameter & Baseline & Post CBT-i & p-value & $\mathbf{N}^{4}$ \\
\hline Sleep onset latency ${ }^{1}$ - minutes & 47.9 & 28.0 & $<0.001$ & 64 \\
\hline $\begin{array}{l}\text { Wake after sleep } \text { onset }^{2} \text { - } \\
\text { minutes }\end{array}$ & 60.7 & 36.2 & $<0.001$ & 57 \\
\hline Number of awakenings & 2.0 & 1.7 & 0.0033 & 61 \\
\hline Total time in bed - minutes & 490.1 & 440.0 & $<0.001$ & 65 \\
\hline Total sleep time - minutes & 382.0 & 378.4 & 0.603 & 66 \\
\hline Sleep efficiency & $78.2 \%$ & $86.1 \%$ & $<0.001$ & 65 \\
\hline $\begin{array}{l}\text { Insomnia severity index (ISI) } \\
\text { score }\end{array}$ & 18.2 & 7.9 & $<0.001$ & 17 \\
\hline
\end{tabular}

1. Sleep onset latency - the time it takes for an individual to fall asleep

2. Wake after sleep onset - the total duration of awakenings during the night after initially falling asleep

3. Sleep efficiency - the proportion of time spent in bed while being asleep

4. Numbers vary as some sleep parameters were not recorded in charts for all patients 
Figure 1 - Patient recruitment

183 patients referred to pharmacist for $C B T-i$

105 patients excluded

19 patients deemed by pharmacist to be poor candidate for CBT-i

32 patients declined CBT-i after initial appointment

54 patients did not return for follow up appointment for unknown reason

78 patients met inclusion criteria ( $\geq 18$ years old, received entire CBT-i intervention from pharmacist) 